\title{
Interaction regimes for oppositely charged plates with multivalent counterions
}

\author{
Fabien Paillusson \\ Department of Chemistry, University of Cambridge, Lensfield Road, Cambridge, CB2 1EW, U.K. \\ Emmanuel Trizac \\ Université Paris-Sud, Laboratoire de Physique Théorique et Modèles Statistiques, UMR CNRS 8626, 91405 Orsay, France
}

(Dated: September 2, 2011)

\begin{abstract}
Within a mean field treatment of the interaction between two oppositely charged plates in a salt free solution, the distance at which a transition from an attractive to a repulsive regime appears can be computed analytically. The mean field description however breaks down under strong coulombic couplings, that can be achieved at room temperature with multivalent counter-ions and highly charged surfaces. Making use of the contact theorem and simple physical arguments, we propose explicit expressions for the equation of state in several situations at short distances. The possibility of Bjerrum pair formation is addressed and is shown to have profound consequences on the interactions. To complete the picture, we finally consider the large distance limit, from which schematic phase diagrams discriminating attractive from repulsive regions can be proposed.
\end{abstract}

PACS numbers:

\section{INTRODUCTION}

$\Gamma \gg 1$ without pair contribution Although it has been less studied than its like-charge counterpart [1-3], the behaviour of two interacting oppositely charged mesoscopic bodies in solution is of importance in various contexts, including colloid physics [4-8], biochemistry related experiment interpretations [9, 10], drug design [11], and structural biology [12]. The simple system of two charged plates with opposite uniform surface charges represents a model of choice that enables one to get analytical results in some limits, and furthermore, that provides a starting point to estimate the interaction energy between two colloids of various geometry [13, 14]. It has been shown that opposite charge repulsion could occur within a mean field (MF) treatment [15, 16]. The physical origin of such a repulsion has been identified to be twofold: a Born repulsion due to short range polarization effects when the solvent has a dielectric constant that significantly exceeds those of the macromolecules [4, 17], and an osmotic repulsion resulting from the trapping of the counter-ions, that ensure electroneutrality between the unequally charged plates [18, 19]. Within a mean-field approach for $q: q$ symmetric solutions (with $q=1$ ), it has been also emphasized recently that the osmotic repulsion may explain how proteins' shape determines their interaction with DNA [20]: the essential physics of the ion mediated interaction between these biomolecules is well captured by a simple two plates model, which opens the way to analytical estimates of the location and depth of the corresponding energy well.

In salt-free solutions with spherical counter-ions of size $b$, the threshold distance $D_{M F}^{*}=h_{M F}^{*}-b$ at which the electrostatic attraction is dominated by the osmotic repulsion for two plates bearing uniform surface charges $\sigma_{1} e$ and $\sigma_{2} e$ (with $\sigma_{1} \sigma_{2}<0$ ), is simply given, within mean-field, by the difference of their respective Gouy-Chapman lengths: $D_{M F}^{*}=\left|\mu_{1}-\mu_{2}\right|$ [19].
These quantities read $\mu_{1(2)}=\left[2 \pi q l_{B}\left|\sigma_{1(2)}\right|\right]^{-1}$, where $l_{B}=e^{2} /\left(4 \pi \varepsilon k_{B} T\right)$ is the Bjerrum length -about $0.7 \mathrm{~nm}$ in water at room temperature- that is defined from temperature $T$ and solvent permittivitty $\varepsilon$. Relying on the Poisson-Boltzmann MF approximation, the previous result only holds provided the Coulombic coupling between counter-ions is not too large. More specifically, this means that the two coupling parameters $\Xi_{1}$ and $\Xi_{2}-$ defined as $\Xi_{i}=2 \pi l_{B}^{2} q^{3}\left|\sigma_{i}\right|$ - should both be small [2, 21]. However, in cases of practical interest with multi-valent counter-ions, the coupling parameter may be large; for instance, converting the charge of double-stranded DNA into an equivalent surface charge, one finds $\Xi \simeq 23$ in water at room temperature with di-valent ions $(q=2)$ and $\Xi \simeq 76$ with $q=3[22$. In this paper, our goal is therefore to study the fate of the attraction/repulsion transition for oppositely charged interfaces, under strong coulombic coupling (large $\Xi$ limit).

For the following discussion, it is instructive to remind the essential features of a single strongly coupled planar double-layer, without added salt (i.e. counter-ions only do ensure electroneutrality) [21 32 . Irrespective of the value of $\Xi$ (from mean-field to strong coupling), the typical distance that counter-ions may explore away from the charged wall is given by the Gouy length $\mu$ defined above. At large $\Xi$ values, the counter-ions form a strongly modulated liquid (if not a true crystal at asymptotically large $\Xi$ ), with a typical distance between ions measured by $a_{\perp}=\sqrt{q / \pi \sigma}$ [23], as required by electroneutrality $\left(\sigma \pi a_{\perp}^{2} \simeq q\right)$. It therefore appears that $\mu \ll a_{\perp}$ when $\Xi \gg 1$, where $a_{\perp}$ measures the size of the correlation hole around each ion. As a consequence, the counterions, that form a strongly correlated liquid parallel to the plate, effectively decouple in the direction perpendicular to the plate, and the leading order profile in the strong-coupling expansion is given by the interactions of individual counter-ions with the confining charged interface [21 32]: this single particle picture simply yields a 
leading exponential counter-ion density profile, with a characteristic length $\mu$. Counter-ions interactions contribute to the sub-leading terms [31], and will not be addressed here: we shall restrict to the low hanging fruits of the single particle viewpoint, that provides the dominant strong-coupling behaviour. We also stress that again for large $\Xi$, we not only have $\mu \ll a_{\perp}$ but also $a_{\perp} \ll q^{2} l_{B}$. More precisely, it is useful to keep in mind the following relations

$$
\frac{a_{\perp}}{\mu \sqrt{2}}=\frac{q^{2} l_{B} \sqrt{2}}{a_{\perp}}=\sqrt{\frac{q^{2} l_{B}}{\mu}}=\sqrt{\Xi},
$$

where the numerical constants are immaterial.

In the following, we shall consider two uniformly charged plates 1 and 2, with respective charge densities $\sigma_{1}<0$ and $0<\sigma_{2}<\left|\sigma_{1}\right|$. Plate 1 is neutralized by counter-ions of valency $q>0$ while $-q$ counter-ions neutralize plate 2 . The corresponding micro-ions remain in the gap of width $h$ between the plates to ensure global electroneutrality (see. Fig,1). Our goal is to characterize the strong coupling regimes, and to infer the equation of state at short distances from the knowledge of ionic density profiles, making repeated use of the contact value theorem 33, 34, that will be reminded in due time. Several situations will be worked out, depending on the formation of Bjerrum pairs $+q /-q$ between oppositely charged micro-ions. In addition to $\Xi_{1}$ and $\Xi_{2}$, the physics of the problem is thus ruled by another coupling parameter, $\Gamma$, to be introduced in section $\amalg \mathrm{A}$ and that quantifies the tendency to form $+q /-q$ pairs. This short range study is developed in sections IIB and IIC. It will be complemented by a large distance analysis in section IID, from which a tentative "phase diagram", allowing to discriminate repulsive from attractive regions, will be put forward. Conclusions will be drawn in section III, and the possible relevance of our approach to weak couplings will be discussed.

\section{STRONG-COUPLING APPROACH FOR OPPOSITELY CHARGED PLATES}

\section{A. Crowding versus pairing}

Whereas previous works pertaining to the strongcoupling limit have been mostly performed in the limit of point counter-ions, it is possible to transpose in some cases the results to the case of finite size ions, essentially by taking $b / 2$ (the ionic radius), as the ion-plate distance of closest approach. For a single plate, the density profiles in the two cases are therefore identical, up to a coordinate shift $z \rightarrow z-b / 2$, where $z$ denotes the distance to the plate. Likewise, in the two plates problem, the plate-plate distance of closest approach is $b$. More precisely, the $b=0$ and $b \neq 0$ cases coincide provided packing effects are negligible (see Fig. 2-left) while increasing ionic size $b$ necessarily leads to a situation where

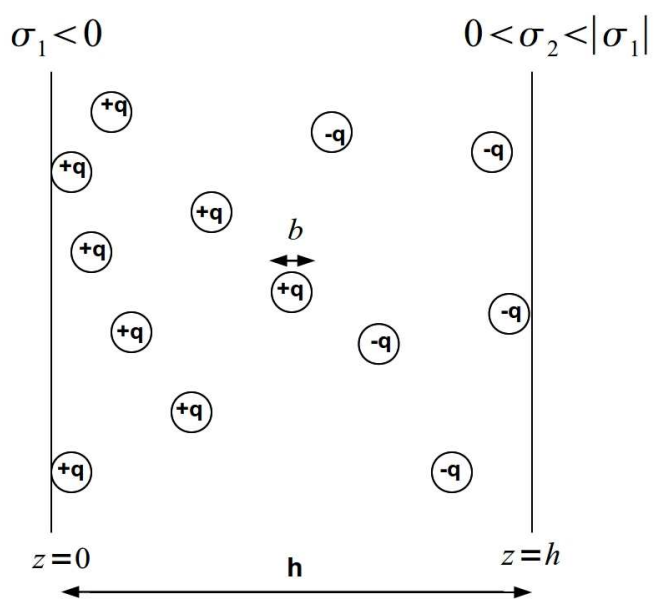

FIG. 1: Schematic view of the two-plates system. Micro-ions are hard spheres of diameter $b$ with charges $+q e$ or $-q e$. The width of the slab between the plates is denoted by $h$, and we define $D$ as $h-b$.
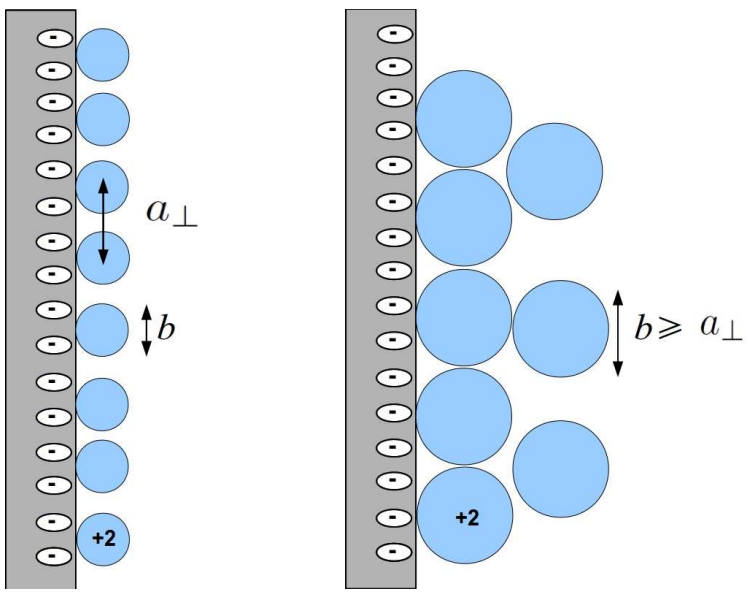

FIG. 2: Two strongly coupled double-layers in different crowding regimes. The left hand side plot shows the uncrowded situation where the finite ionic size does not perturb the point-counter-ion predictions, while on the other hand, the right hand side plot is for a case where hard core effects lead to crowding, with bi or multi-layers of counter-ions in the vicinity of the plate. For this example to be meaningful, we set $q=+2$ for the valency of the counterions.

$b$ becomes of the order of $a_{\perp}$, so that the double-layer can no longer accommodate a mono-layer of counter-ions (see Fig. 2 -right). Understanding the behaviour of strongly coupled and crowded double-layers is an open problem that lies beyond the scope of the present work, so that we will restrict to the cases where $b<a_{\perp}$, i.e. to not too big micro-ions. This requirement should be enforced for both plates: $b<a_{\perp}^{(1),(2)}$.

In addition to crowding, micro-ion pairing may take place in the two plates problem [2, 35], see Fig.3. The tendency for $+q$ and $-q$ micro-ions to form neutral pairs 


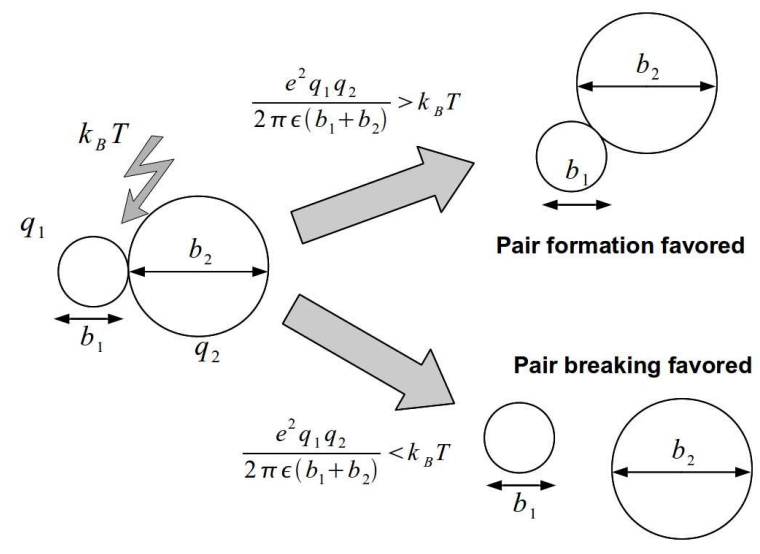

FIG. 3: Pair breaking/formation mechanism. Starting from a contact configuration for two ions of opposite charges $q_{1} e$ and $-q_{2} e$ on the left hand side, the tendency to remain in this configuration, or, on the contrary, to break the pair, is given by comparing the electrostatic loss to the thermal energy.

at $T \neq 0$ is quantified by the ratio between the direct electrostatic interaction at close contact and $k_{B} T$ i.e. $\Gamma=$ $q^{2} l_{B} / b$. Interestingly, keeping in mind the "no-crowding" condition sketched above $\left(b<a_{\perp}^{(1),(2)}\right)$, we get the inequality $2 \Gamma^{2}>\Xi\left(\right.$ where $\left.\Xi=\max \left(\Xi_{1}, \Xi_{2}\right)=\Xi_{1}\right)$, so that the possible values $\Gamma$ can take are bounded from below by $\sqrt{\Xi / 2}$. Consequently, strongly coupled uncrowded double-layers lead to the important formation of Bjerrum pairs (large $\Gamma$ ). We nevertheless start by considering the rather narrow region where $3 \lesssim \sqrt{\Xi / 2}<\Gamma<10$ in which pair formation can be neglected. The above constraint translates into $20<\Xi<200$, where $\Xi$ is large enough to allow for a strong coupling analysis in due form to unveil the main features. A more quantitative description presumably requires, especially at the smaller $\Xi$-values involved, an intermediate approach interpolating between the mean-field and strong-coupling limits [36 39].

\section{B. Small separation distances without pair formation}

We define in the subsequent analysis $D$ as the shifted distance between the plates : $D=h-b$. The first situation addressed is that where Bjerrum pair formation can be neglected, which is the assumption made in [25]. Under strong coupling, if $D<a_{\perp}^{(1)}$ (which implies that $D<a_{\perp}^{(2)}$ since $\left.\sigma_{2}<\left|\sigma_{1}\right|\right)$, the single particle picture where each micro-ion only interacts with both plates and not with its fellow micro-ions is valid. A tagged microion feels an electric field $-2 \pi\left(\left|\sigma_{1}\right|+\sigma_{2}\right) \hat{z} / \varepsilon$ where $\hat{z}$ is a unit vector along the $z$ direction, hence a linear potential in $z$. The corresponding number densities $n_{+}(z)$ and $n_{-}(z)$ for both $+q$ and $-q$ species follow then a simple
Boltzmann law:

$$
n_{ \pm}(z)=n_{ \pm}^{(0)} e^{\mp \tilde{z}}
$$

where $n_{+}^{(0)}$ and $n_{-}^{(0)}$ are two normalization constants, and where we introduced the reduced distance to plate 1 ,

$$
\tilde{z}=z / \lambda \quad \text { with } \quad \frac{1}{\lambda}=\frac{1}{\mu_{1}}+\frac{1}{\mu_{2}} .
$$

The two factors $n_{ \pm}^{(0)}$ can be determined from the electroneutrality conditions:

$$
\begin{gathered}
q \int_{b / 2}^{h-b / 2} d z n_{+}^{(0)}=\left|\sigma_{1}\right| \\
q \int_{b / 2}^{h-b / 2} d z n_{-}^{(0)}=\sigma_{2},
\end{gathered}
$$

so that

$$
\begin{aligned}
& n_{+}(\tilde{z})=\frac{\left|\sigma_{1}\right| e^{\tilde{b} / 2-\tilde{z}}}{q \lambda\left(1-e^{\tilde{b}-\tilde{h})}\right.} \\
& n_{-}(\tilde{z})=\frac{\sigma_{2} e^{-\tilde{b} / 2+\tilde{z}}}{q \lambda\left(e^{-\tilde{b}+\tilde{h}}-1\right)} .
\end{aligned}
$$

The expression for the reduced pressure

$$
\Pi=2 \pi l_{B} q^{2} \mu_{1}^{2} \beta P=\frac{\beta P}{2 \pi l_{B} \sigma_{1}^{2}}
$$

directly follows from the contact value theorem, which yields the pressure $P$ in the form [33, 34]:

$$
\begin{aligned}
P & =n_{+}\left(\frac{b}{2}\right)+n_{-}\left(\frac{b}{2}\right)-2 \pi l_{B} \sigma_{1}^{2} \\
& =n_{+}\left(h-\frac{b}{2}\right)+n_{-}\left(h-\frac{b}{2}\right)-2 \pi l_{B} \sigma_{2}^{2}
\end{aligned}
$$

Consequently, we have

$$
\Pi(\widetilde{D})=\zeta \operatorname{coth}\left(\frac{\widetilde{D}}{2}\right)+\frac{1}{2}\left(1+\zeta^{2}\right)\left[\operatorname{coth}\left(\frac{\widetilde{D}}{2}\right)-1\right]
$$

where we introduced the charge ratio $\zeta=\sigma_{2} /\left|\sigma_{1}\right|$. Equation (11) is independent of the plate (1 or 2$)$ where the contact theorem is applied, which provides a consistency test for the argument. In other words, the pressure $P$ is invariant under the change $\zeta \rightarrow 1 / \zeta$, so that the reduced pressure should change according to $\Pi \rightarrow \Pi \zeta^{-2}$ when $\zeta \rightarrow \zeta^{-1}$. This property can be checked directly on Eq. (11). More importantly, expression (11) is positive for $D>0$, for all values of the charge ratio, see Fig. 4. Therefore, the interaction between two oppositely charged plates is always repulsive at short distances in this regime. The physical mechanism behind this repulsive behaviour is the following. Compared to the large distance limit where $n_{+}(b / 2) \simeq 2 \pi l_{B} \sigma_{1}^{2}$, as follows from Eq. (9) and the fact that both the pressure and 
$n_{-}(b / 2)$ vanish, bringing the plates at short distances where $D<a_{\perp}^{(1)}$ enhances the electric field felt by $+q$ micro-ions, which has the result to increase their density at contact with plate 1 . Invoking again the contact theorem (9), the consequence is that $P>0$. The interactions between $+q$ and $-q$ micro-ions could counterbalance this effect, but these interactions have been discarded here, with the neglect of Bjerrum pair formation. We will see below that $+q /-q$ interactions, when relevant, completely change the phenomenology.

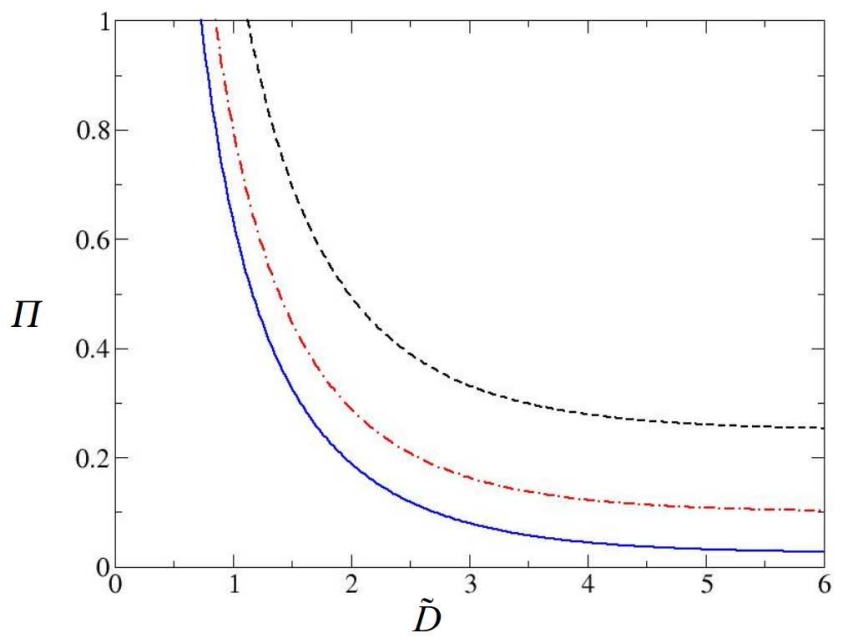

FIG. 4: Plot of the reduced pressure following from the shortdistance equation of state (11), as a function of rescaled distance $\widetilde{D}$, for several values of $\zeta=\sigma_{2} /\left|\sigma_{1}\right|: \zeta=0.25$ (dashed line), $\zeta=0.1$ (dot-dashed line) and $\zeta=0.025$ (solid line). The short-distance requirement $D<a_{\perp}^{(1)}$ (the so-called RouzinaBloomfield criterion [23]), translates into $\widetilde{D}<\Xi^{1 / 2}$.

Three remarks are in order here. 1) We see that the reason for observing repulsive behaviour (enhanced electric field acting on a micro-ion within the single particle picture) is the same as that leading to attraction in the like-charged case (decreased electric field, with a corresponding decrease of micro-ionic density at contact; this effect is most pronounced in the symmetric case where $\sigma_{1}=\sigma_{2}$, for which the electric field vanishes and the micro-ion densities is uniform in the $z$ direction). 2) The possibility of attraction under strong coupling reported in 25] for oppositely charged plates stems from the fact that only one type of micro-ion was considered in 25]. This results in a smaller amount of counter-ions (per unit surface), compared to that which is necessary to neutralize the isolated plate, with a concomitant decrease of contact ionic density, which opens the way to a possible attraction. The physical situation considered in [25] thus differs from the one under study here. 3) Our finding $P>0$ relies on the condition $D<a_{\perp}^{(1)}$. At large distances, we should have $P<0$ since a mean-field scenario is then expected to prevail [24, 36, 40, 41]. We will come back to this point in section IID.

\section{Small separation distances with pair formation}

We now turn to the case where $1 \ll \sqrt{\Xi / 2}<\Gamma$, with a strong tendency for two micro-ions to form a neutral pair. While pair formation is unlikely as long as the two condensed micro-ion layers from each plate do not overlap, it turns important at smaller separations. By "pair formation", we loosely refer here to the more or less complex structures, or aggregates, that may form from the association of several of individual pairs. Pairs may indeed exist in the form of well defined entities, but may also self assemble into chains, see e.g. Ref. [42], or into more complex structures (regular or empty crystals) uncovered in a related context in Ref. [43]. The corresponding aggregates are electrically neutral, with number of Bjerrum pairs involved per unit area limited by the less abundant species of micro-ion, i.e. the counter-ions of the plate 2 . Therefore, the aggregate surface density is bounded from above by $\sigma_{2} / q$. These aggregates coexist with a strongly correlated Wigner-like crystal made up of the remaining majority species. In this work, we did not attempt at a precise evaluation of the aggregate, or "pairs" contribution $P_{a g g}$ to the total interplate pressure $P$, but instead, we considered two limiting cases, where we bound $P_{a g g}$ from below by 0 (see section IIC 1), and from above by $k T \sigma_{2} /(q D)$ (see section IC 2). The latter bound corresponds to a density of aggregates, that are neutral entities, equal to $\sigma_{2} /(q D)$, that is, to the maximum mean density of possible pairs. Any self-assembly of the pairs in a more complex architecture leads to a decrease of that density. We now investigate separately these two limiting cases.

\section{Without the osmotic contribution from the pairs}

With only positive counter-ions in the system, the typical lateral distance becomes $A_{\perp}=\sqrt{q / \pi\left(\left|\sigma_{1}\right|-\sigma_{2}\right)}$ and for separation distances $D$ less than $A_{\perp}$, the single particle picture holds, and yields the micro-ionic density $n_{+}(z)$ in a form similar to (2):

$$
n_{+}(z)=n_{+}^{(1)} e^{-\tilde{z}}
$$

where $n_{+}^{(1)}$ is a positive constant. As for $n_{+}^{(0)}$, the $n_{+}^{(1)}$ prefactor can be determined using the electroneutrality condition:

$$
q \int_{b / 2}^{h-b / 2} d z n_{+}(z)=\left|\sigma_{1}\right|-\sigma_{2}
$$

and the ion density then reads:

$$
n_{+}(z)=\frac{\left|\sigma_{1}\right|-\sigma_{2}}{q \lambda\left(1-e^{\tilde{b}-\tilde{h}}\right)} e^{\tilde{b} / 2-\tilde{z}} .
$$

In a first step, we do not consider the contribution of Bjerrum pairs to the total pressure (we therefore bound 
from below the term $P_{a g g}$ by 0 ). In doing so, the pressure at a given reduced separation distance $\widetilde{D}$ can again be found by means of the contact value theorem, with only one species of micro-ions: $\beta P=n_{+}(b / 2)-2 \pi l_{B} \sigma_{1}^{2}$, so that

$$
\Pi(\widetilde{D})=-\frac{1}{2}\left(1+\zeta^{2}\right)+\frac{1}{2}\left(1-\zeta^{2}\right) \operatorname{coth}\left(\frac{\widetilde{D}}{2}\right)
$$

We recover the same expression as in [25], from a "mechanical" (contact theorem) instead of "energy" route. Unlike Eq.(11), Eq.(15) does not have a definite sign and as a consequence, the interaction is attractive at large distances: there exists a threshold

$$
D_{0}^{*}=2 \lambda \ln \left(\left|\sigma_{1}\right| / \sigma_{2}\right)
$$

below which the interaction becomes repulsive $(\Pi>0)$, see Fig. 5. We note from Eq. (16) that attraction prevails until $D \rightarrow 0$ when $\sigma_{2} \rightarrow\left|\sigma_{1}\right|$ and we add that as long as micro-ions (even in small amount) remain between the plates as required by electroneutrality, the corresponding entropy cost for confinement makes the pressure diverge (hence positivity) for $D \rightarrow 0$. Only for $\zeta=1$, i.e. $\sigma_{2}=-\sigma_{1}$ would micro-ion total density vanish, which leaves two oppositely charged plates interacting without any screening. In that specific case, the interaction is obviously attractive until close contact $h=0$. On the other hand, in the large $\widetilde{D}$ limit -with nevertheless the $D<A_{\perp}$ requirement enforced-, and for any value of $\zeta$, one can immediately find the pressure from the contact theorem applied at plate 2 : for large $\widetilde{D}$, the positive micro-ions are expelled from the vicinity of the positive plate, which means that the contact density $n_{+}(h-d / 2)$ vanishes and that $\beta P \rightarrow-2 \pi l_{B} \sigma_{2}^{2}$. In terms of rescaled pressure, we then have $\Pi \rightarrow-\zeta^{2}$, which is indeed observed in Fig. 5 .

\section{With the osmotic contribution from the pairs}

We now include the pairs contribution to the equation of state, through the upper bound $\sigma_{2} /(q D)$ alluded to above. We then get

$$
\Pi=\frac{(1+\zeta) \zeta}{\widetilde{D}}-\frac{1}{2}\left(1+\zeta^{2}\right)+\frac{1}{2}\left(1-\zeta^{2}\right) \operatorname{coth}\left(\frac{\widetilde{D}}{2}\right)
$$

Clearly, compared to expression (15), the effect of this osmotic contribution is to increase the threshold value where repulsion $(\Pi>0)$ can be observed. The two limiting behaviours, Eq. (15) and Eq. (17), are sketched in Fig. 5. The corresponding values of the thresholds $\widetilde{D}_{0}^{*}$ and $\widetilde{D}_{1}^{*}$ are indicated. These two quantities are plotted in Fig. 6] as a function of charge asymmetry, together with the analytical estimation of $\widetilde{D}_{1}^{*}$ obtained as follows. If $\widetilde{D}$ is large enough, Eq. (17) simplifies to:

$$
\Pi \simeq-\zeta^{2}+\frac{(1+\zeta) \zeta}{\widetilde{D}} .
$$

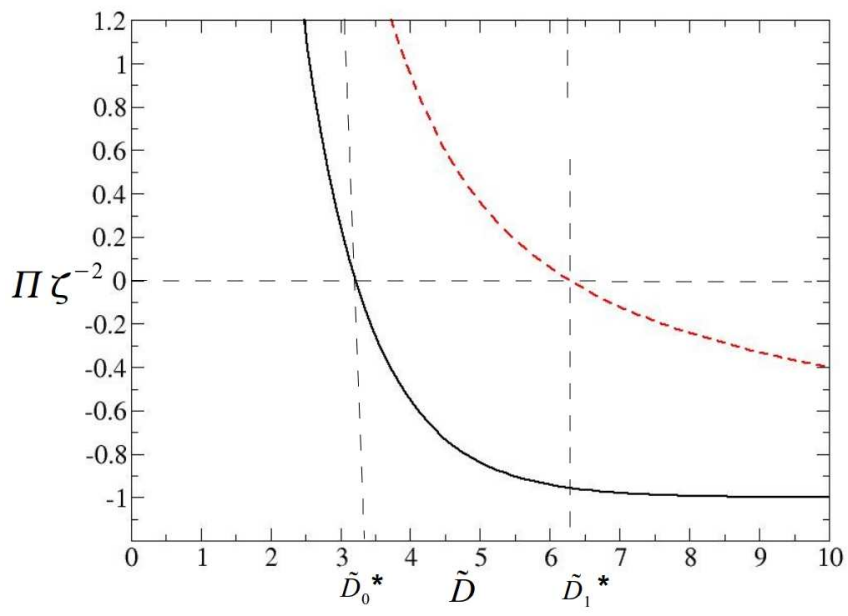

FIG. 5: Pressure curves from Eqs. (15) (solid line) and (17) (dashed line) for $\zeta=0.2$.

This expression can now exhibit a repulsive behaviour below $\widetilde{D}=(1+\zeta) / \zeta$ i.e.

$$
D_{1}^{*} \simeq \mu_{2}
$$

It can be seen that this approximation (dashed line) is in fair agreement with the root of Eq. (17) found numerically (dotted line), in the whole available range.

The single particle picture invoked here relies on two assumptions. First, $\Gamma$ should be large, to have pair formation. Second, the (shifted) distance $D$ between the plates should not exceed $A_{\perp}$, see the upper dot-dashed line in Fig. 6. Making use of approximation (19), this means $1 /\left(2 \Xi_{2}+1\right)<\zeta$. For smaller values of $\zeta$, the analysis is significantly more complex (loss of the single particle view-point). Hence, if $\zeta>1 /\left(2 \Xi_{2}+1\right)$ the interaction is repulsive at short distances and then turns attractive at intermediate distances, while if $\zeta<1 /\left(2 \Xi_{2}+1\right)$, Eq.(18) does not lead to any transition between repulsion and attraction, and is always repulsive in its range of validity, as was the case for Eq.(11). Of course, for large $\Xi_{2}$, the threshold $\left(2 \Xi_{2}+1\right)^{-1}$ is small, so that extremely asymmetric cases only (very low $\zeta$ are not covered by our analysis.

\section{Large separation distances}

Our analysis has so far been restricted to short distance expansions. We are now interested in large distance asymptotics and in attempting to match the short and large distance behaviours. In doing so, we will discuss qualitatively an attraction/repulsion transition of an "effective" mean-field type, which leads to reentrant attraction as the distance between the two plates is varied from infinity to close contact. 


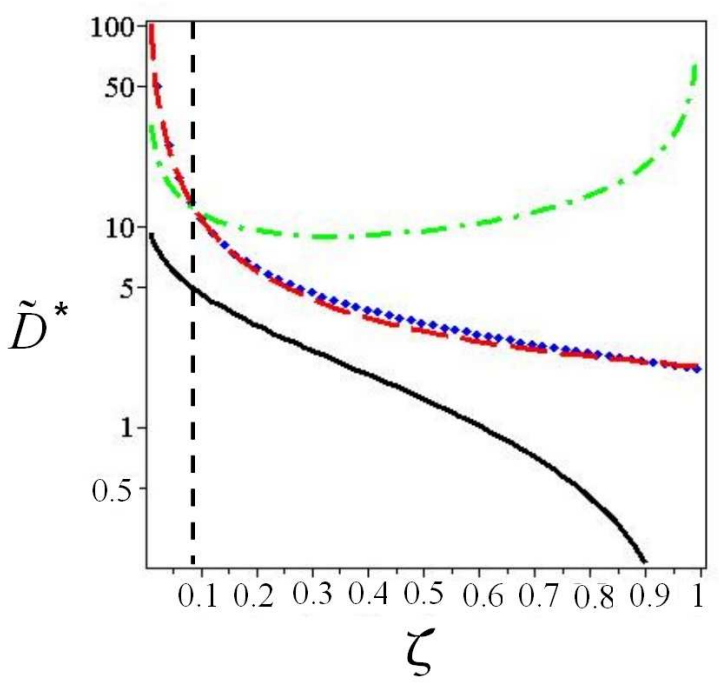

FIG. 6: Reduced threshold distance discriminating (strongcoupling) attraction from repulsion, as a function of $\zeta$, in semi-log scale. The solid line shows $\widetilde{D}_{0}^{*}$ obtained from (15), the dotted line is for $\widetilde{D}_{1}^{*}$, the exact root of Eq. (17), and the dashed line displays the approximation (19). The upper dotdashed line shows $\tilde{A}_{\perp}$, in the particular case $\Xi_{2}=25$ : our approach is meaningful for $\widetilde{D}<\tilde{A}_{\perp}$ only, which translates into $\zeta>1 /\left(2 \Xi_{2}+1\right)$, see text. Here, $1 /\left(2 \Xi_{2}+1\right) \simeq 0.02$.

\section{Crossover between strong-coupling and mean-field regions for one plate}

We will assume first that a given strongly-coupled plate (having thus a large $\Xi_{i}$ ), can be effectively described by mean-field theory, at sufficiently large distances $z$. This "common wisdom" stems on the remark that for large $z$, the typical distance between counter-ions becomes large, which leads to a low coupling regime [24, 36, 40, 41]. It should be emphasized though that the above point of view, that predicts a large $z$ density decay in $1 / z^{2}$, is incorrect in two dimensions, as shown in a recent work 31]. The present study pertains to three dimensional systems, so that we nevertheless expect for a single plate the crossover scenario discussed in Ref. [41], and summarized in Fig. 7. In essence, the density is expected to decrease exponentially at short distances, and algebraically at large distances: beyond a distance $\delta$ from the plate, the counter-ion density $n$ is simply given by the solution of the nonlinear Poisson-Boltzmann (PB) equation:

$$
n(\mathfrak{z})=\frac{1}{2 \pi l_{B} q^{2}\left(\mathfrak{z}+\mu^{\text {eff }}\right)^{2}}
$$

where $\mathfrak{z} \equiv z-b / 2$ and where $\mu^{\text {eff }}$ is an effective Gouy-Chapman length characterizing this long range behaviour. Following [41], one can match the two regimes by assuming that the condensed counter-ion layer forms a 2D One Component Plasma and by applying a meanfield approximation for the dilute layer. Equating the two corresponding chemical potentials yields

$$
n(\delta)=n_{s c} e^{-\beta\left|\varepsilon_{c}\right|}
$$

where $n_{s c}$ is a $\delta$-related average density in the condensed layer and $\beta \varepsilon_{c}(\Xi) \approx-1.56 \sqrt{\Xi}$ is the contribution to the $2 \mathrm{D}$ one component plasma chemical potential that stems from the correlations between the counter-ions [2, 24]. Extrapolating the validity of Eq.(20) to $\delta \rightarrow b / 2$ and assuming that in such a situation, $n_{s c}$ is well approximated by the average density over the characteristic length $l_{s c}$, we arrive at [41]:

$$
n(\mathfrak{z}=0)=\frac{|\sigma|}{q l_{s c}}\left[1-e^{-l_{s c} / \mu}\right] e^{-1.56 \sqrt{\Xi}} .
$$

Equation (22) is nothing but the density that the meanfield profile, valid at large distances from the plate, would have if extrapolated at $\mathfrak{z}=0$, and is therefore not the real density at the plate. However, invoking Eq.(20), it allows one to estimate the effective Gouy-Chapman length $\mu^{\text {eff }}$ corresponding to the charged plate dressed by a condensed counter-ion layer, which will prove useful in the following. For the subsequent quantitative discussion, we shall take the value $l_{s c} \approx 3.6 \mu$, already used in [41].

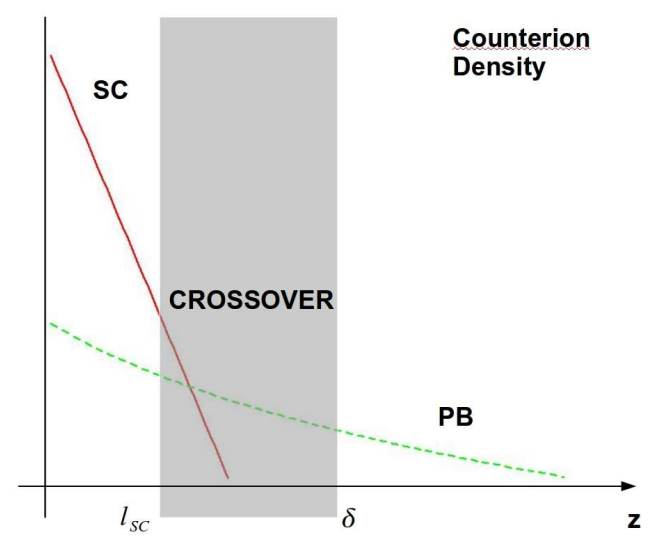

FIG. 7: Crossover scheme: following Ref. [41], schematic representation in semi-log scale of the crossover between strong coupling (SC) and mean-field regimes for the counter-ion density in the vicinity of a single highly charged plate. The solid curve represents the exponential decay expected close to the plate while the dashed curve stands for the algebraic decay expected at large distances where the Poisson-Boltzmann theory (PB) should hold. The shaded region corresponds to the crossover between these two regimes: $l_{s c}$ is the distance beyond which the exponential decay no longer holds and $\delta$ is the distance to the plate beyond which the PB profile is expected to be valid.

\section{Application to the two plates problem}

When the separation distance between plates 1 and 2 is decreased from infinity, the first inter-plate weak interaction regime is expected to be of mean-field type, so 
that the presumably large distance attraction may turn into repulsion at a distance $D_{M F}^{*}=\left|\mu_{1}^{\text {eff }}-\mu_{2}^{\text {eff }}\right|$. In this picture, the distance is varied at constant effective GouyChapman lengths $\mu_{1}^{\text {eff }}$ and $\mu_{2}^{\text {eff }}$ given by:

$$
\mu_{i}^{\mathrm{eff}}=1.92 \mu_{i} e^{0.78 \sqrt{\Xi_{i}}}, i=1,2 .
$$

If $\left|\mu_{1}^{\text {eff }}-\mu_{2}^{\text {eff }}\right|$ is significantly larger than the characteristic thresholds obtained in the previous subsections, we should have the following "reentrant" sequence \{attraction $\rightarrow$ repulsion $\rightarrow$ attraction $\rightarrow$ repulsion $\}$ as $D$ decreases. The first transition is described by a meanfield argument, and the last one by strong-coupling considerations, but the intermediate transition \{repulsion $\rightarrow$ attraction $\}$ occurs in a crossover region that resists our theoretical understanding, and where additional \{repulsion $\rightarrow$ attraction $\}$ transitions might take place. A related question deals with the lower bound for the distance $D_{\text {bound }}$ below which the mean-field profiles are no longer accurate. For the sake of completeness, we will consider below that $D_{\text {bound }}=a_{\perp}^{(1)}+a_{\perp}^{(2)}$. Depending on the respective surface charge densities $\sigma_{1}$ and $\sigma_{2}$, we can then discriminate between two distinct situations:

- $\left|\mu_{1}^{\text {eff }}-\mu_{2}^{\text {eff }}\right|<D_{\text {bound }}$. The interaction between the two plates is always attractive at large distances (mean-field regime), and then at short separation distances, the strong-coupling phenomenology described in the first part of the paper prevails.

- $\left|\mu_{1}^{\text {eff }}-\mu_{2}^{\text {eff }}\right|>D_{\text {bound }}$. There is then already a transition between attraction and repulsion in the mean-field regime. By decreasing further the distance $D$ and entering the short distance limit, one should observe another attractive range, as expressed in Eq. (15) for instance, before repulsion sets in at even smaller separations.

More complicated scenarios could be envisioned, but we summarize in Fig. 8 the simplest possible, and provide a phase diagram obtained when considering that the Bjerrum pairs do contribute to the pressure, as in section II C2. We note that, for the parameters chosen, there is a reentrant behaviour observed with respect to the separation distance, in a large fraction of the $(\zeta, D)$ plane, more specifically when $\zeta<0.5$ (this threshold depends on the value of $\Xi_{2}$ chosen, and increases with $\Xi_{2}$ ). We remind that the bottom part of the diagram, more specifically for $\zeta<1 /\left(2 \Xi_{2}+1\right)$, corresponds to a region where our arguments do not apply, as discussed in section $\amalg$ C2. In this region, our short scale analysis provides an "all repulsive" behaviour, and we may then speculate that repulsion persists up to the effective mean-field threshold indicated by the asterisks, which corresponds to large distances, on the order of $100 q^{2} l_{B}$ or more.

\section{DISCUSSION AND CONCLUSION}

In this paper, we have analyzed the interaction of two oppositely charged parallel interfaces, each neutralized

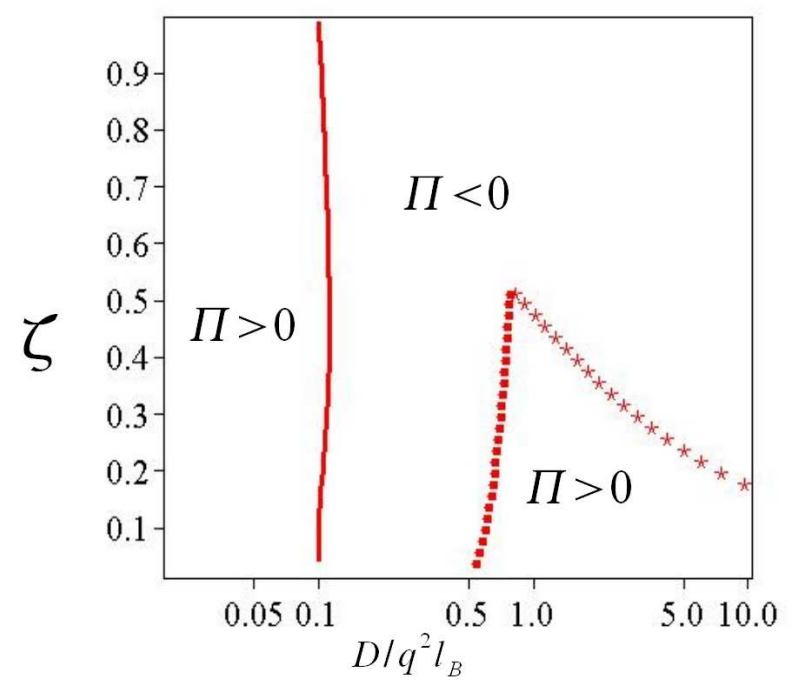

FIG. 8: Sketch of attractive and repulsive regimes, as a function of the ratio $\zeta=\sigma_{2} /\left|\sigma_{1}\right|$ and the distance $D$ (in $\log$ scale). The repulsive island on the left hand side, delimitated by a continuous line, shows $D_{1}^{*}$ where the pressure in Eq. (17) vanishes. The other repulsive region on the right hand side, delimitated by a dotted line (star symbols), shows $D_{M F}^{*}=\left|\mu_{1}^{\text {eff }}-\mu_{2}^{\text {eff }}\right|$, where the effective Gouy-Chapman lengths are given by Eqs.(23). These data are displayed provided they satisfy the constraint $D_{M F}^{*}>D_{\text {bound }}=a_{\perp}^{(1)}+a_{\perp}^{(2)}$. Likewise, the left boundary for this mean-field repulsive island has been taken to be $D_{\text {bound }}$, that is shown with a dotted line (square symbols). Here, we have taken $\Xi_{2}=10$.

by its own counter-ions, without other micro-ions involved (salt-free case, but with two species of microions, of opposite signs). We have shown that a repulsive behaviour, quite expectedly, is always present at short enough separations; it simply stems from the diverging entropy cost for confining micro-ions in a slab of vanishing extension. Our analysis completes the known Poisson-Boltzmann phenomenology by investigating the case of strong coulombic couplings. Short distance expansions reveal that depending on the formation of Bjerrum pairs between the oppositely charged micro-ions, an attractive regime may or may not be observed. By formation of pairs, we understand here the wealth of different self-assembly scenarios where the pairs may further associate into more complex objects, such as chains or various crystals [42, 43]. We did not attempt at a precise evaluation of the corresponding contribution to the pressure - a particularly demanding task- but rather, we analyzed limiting cases where this unknown contribution is bounded by reasonable values, see section IIC. We have supplemented our short distance analysis by a more speculative investigation of the large distance behaviour, from which a "phase diagram" was put forward, with reentrant features between attraction and repulsion as the distance $D$ between the plates is varied. The experimental observation of such features would imply that 
other sorts of interactions, such as van der Waals, do not modify the main effects uncovered.

In our approach, single particle arguments play a crucial role and allow us to compute the density of microions, from which the equation of state follows. These single particle arguments, however, are a priori not restricted to strongly coupled interfaces, but can equally be invoked when the coupling parameters $\Xi_{1}$ and $\Xi_{2}$ are small (see e.g. section 3.3 of Ref 44] and in particular Fig. 16 for simulation results backing up this statement in the like-charge case $\sigma_{1}=\sigma_{2}$ ). Indeed, when $D$ becomes smaller than the characteristic lateral distance $a_{\perp}$ between ions, these ions effectively decouple and feel the external potential of the plates only (we are here concerned with the ionic density dependence on the $z$ coordinate, perpendicular to the plate; in the transverse direction, parallel to the plate, a correlation hole remains around each particle, of typical size $q^{2} l_{B}$ ). As a consequence, the pressures given by Eqs. (11), (15) and (17) still hold with the same range of validity, for $\Xi_{i} \rightarrow 0$. In the corresponding distance range, the Poisson-Boltzmann results break down due to discreteness effects [it is therefore essential here to make a clear distinction between Poisson-Boltzmann theory, and the low $\Xi$ limit of the original model dealing with discrete particles ; Poisson-Boltzmann approach considers from the outset continuous density fields, and can therefore not be expected to hold at separation distances such that discreteness effects do matter, i.e. when $D<a_{\perp}$; the ade- quacy of Poisson-Boltzmann to describe the low $\Xi$ physics should then be understood as a statement which excludes a small range of short separations $D]$. From the analysis of section [I], we learn that when Bjerrum pair formation can be neglected, the threshold distance $D^{*}$ where repulsive behaviour sets in is still given by the PoissonBoltzmann result $\left|\mu_{2}-\mu_{1}\right|=\mu_{2}-\mu_{1}$, provided this length is larger than both characteristic distances $a_{\perp}^{(1)}$ and $a_{\perp}^{(2)}$. In the opposite case, when $\left|\mu_{2}-\mu_{1}\right|<\inf \left(a_{\perp}^{(1)}, a_{\perp}^{(2)}\right)$, we may speculate that $D^{*}$ lies between $a_{\perp}^{(1)}$ and $a_{\perp}^{(2)}$, since the single particle argument which holds at smaller separations leads to repulsion, while the Poisson-Boltzmann theory yields attraction at larger separations 20]. If on the other hand, Bjerrum pairs form and contribute to the pressure through their mean density (see section II C2), we have seen that $D^{*}=\mu_{2}$, which is thus larger than the Poisson-Boltzmann result $\mu_{2}-\mu_{1}$. However, this result only holds provided $\zeta>\left(1+2 \Xi_{2}\right)^{-1} \simeq 1$ (we are still considering the low $\Xi_{i}$ limit). Given that $\zeta \leq 1$ by definition (i.e. $\sigma_{2}<\left|\sigma_{1}\right|$ ), we see that here, the single particle picture does not apply up to $\mu_{2}$ (except in a small $\zeta$ region close to 1 ), which means that $D^{*}$ is larger than $A_{\perp}=\left[\pi\left(\left|\sigma_{1}\right|-\sigma_{2}\right)\right]^{-1 / 2}$. It can be checked that generically, this length is smaller than the PoissonBoltzmann prediction $\mu_{2}-\mu_{1}$, except again in a small $\zeta$-region around unity.

Acknowledgment. This work has been partly supported by the EPSRC grant $N^{\circ} E P / I 000844 / 1$.
[1] J.-P. Hansen and H. Löwen, Annu. Rev. Phys. Chem. 51, 209 (2000).

[2] Y. Levin, Rep. Prog. Phys. 65, 1577 (2002).

[3] R. Messina, J. Phys.: Condens. Matter 21, 113102 (2009).

[4] N. Ben-Tal, J. Phys. Chem. 99, 9642 (1995).

[5] J. Z. Wu, D. Bratko, H. W. Blanch, and J. M. Prausnitz, Phys. Rev. E 62, 5273 (2000).

[6] E. J. Tull, P. Bartlett, and K. R. Ryan, Langmuir 23, 7859 (2007).

[7] J. Jens Rydén, M. Ullner, and P. Linse, J. Chem. Phys. 123, 034909 (2005)

[8] V. Dahirel and J.-P. Hansen, J. Chem. Phys. 131, 084902 (2009).

[9] B. Jonsson and J. Stahlberg, Col. Surf. B 14, 67 (1999).

[10] S. Bigdeli, A. H. Talasaz, P. Stahl, H. Persson, M. Ronaghi, R. W. Davis, and M. Nemat-Gorgani, Biotec. Bioen 100, 19 (2008).

[11] J.-R. Morones et al., Nanotechnology 16, 2346 (2005).

[12] S. Jones, H. Shanahan, H. Berman, and J. Thornton, Nucleic Acids Research 31, 7189 (2003).

[13] J. Vervey and J. T. G. Overbeek, Theory of the Stability of Lyophobic colloïds (Elsevier: Amsterdam, 1948).

[14] S. Bhattacharjee and M. Elimelech, J.Colloid Inteface Sci. 193, 273 (1997).

[15] V. A. Parsegian and D. Gingell, Biophys. J. 12, 1192 (1972).

[16] H. Ohshima, Colloid and Polymer Sci. 253, 150 (1975).
[17] P. Sens and J.-F. Joanny, Phys. Rev. Lett. 84, 4862 (2000).

[18] D. Ben-Yaakov, Y. Burak, D. Andelman, and S. Safran, EPL 79, 48002 (2007).

[19] F. Paillusson, M. Barbi, and J.-M. Victor, Mol. Phys. 107, 1379 (2009).

[20] V. Dahirel, F. Paillusson, M. Jardat, M. Barbi, and J.-M. Victor, Phys. Rev. Lett. 102, 228101 (2009).

[21] R. Netz, Eur. Phys. J. E 5, 557 (2001).

[22] A. Naji, S. Jungblut, A. Moreira, and R. Netz, Physica A 352, 131 (2005).

[23] I. Rouzina and V. Bloomfield, J. Phys. Chem. 100, 9977 (1996).

[24] B. I. Shklovskii, Phys. Rev. E 60, 5802 (1999).

[25] M. Kanduč, M. Trulsson, A. Naji, Y. Burak, J. Forsman, and R. Podgornik, Phys. Rev. E 78, 061105 (2008).

[26] Y. S. Jho, M. Kanduč, A. Naji, R. Podgornik, M. W. Kim, and P. A. Pincus, Phys. Rev. Lett. 101, 188101 (2008).

[27] D. Dean, R. Horgan, A. Naji, and R. Podgornik, J. Chem. Phys. 130, 094504 (2009).

[28] M. Kanduč, A. Naji, J. Forsman, and R. Podgornik, J. Chem. Phys. 132, 124701 (2010).

[29] M. Hatlo and L. Lue, EPL 89, 25002 (2010).

[30] M. Kanduč, A. Naji, J. Forsman, and R. Podgornik, arXiv:1101.1362 (2011).

[31] L. Šamaj and E. Trizac, Eur. Phys. J. E 34, 20 (2011).

[32] L. Šamaj and E. Trizac, Phys. Rev. Lett. 106, 078301 
(2011)

[33] D. Henderson and L. Blum, J. Chem. Phys. 69, 5441 (1978).

[34] H. Wennerström, B. Jönsson, and P. Linse, J. Chem. Phys. 76, 4665 (1982).

[35] J. Zwanikken and R. van Roij, J.Phys.: Condens. Matt. 21, 4241102 (2009).

[36] Y. Burak, D. Andelman, and H. Orland, Phys. Rev. E 70, 016102 (2004).

[37] Y.-G. Chen and J. Weeks, Proc. Nat. Acad. Sci. USA 103, 7560 (2006).

[38] C. D. Santangelo, Phys. Rev. E 73, 041512 (2006).
[39] S. Buyukdagli, M. Manghi, and J. Palmeri, Phys. Rev. Lett. 105, 158103 (2010).

[40] H. Boroudjerdi, Y.-W. Kim, A. Naji, R. Netz, X. Schlagberger, and A. Serr, Phys. Rep. 416, 129 (2005).

[41] A. dos Santos, A. Diehl, and Y. Levin, J. Chem. Phys. 130, 124110 (2009).

[42] J. J. Weis and D. Levesque, Phys. Rev. Lett. 71, 2729 (1993).

[43] L. Assoud, R. Messina, and H. Löwen, EPL 89, 36001 (2010).

[44] A. Moreira and R. Netz, Eur. Phys. J. E 8, 33 (2002). 